$\xi_{p}$

\title{
Vegetation Monitoring Using UAV : a Preliminary Study
}

\author{
Soon Eng L ${ }^{1 *}$, Rozita Ismail, Wahidah Hashim², Rajina R. Mohamed ${ }^{3}$, Aslina Baharum ${ }^{4}$ \\ College of Computer Science and Information Technology, University Tenaga Nasional \\ Faculty of Computing and Informatics, Universiti Malaysia Sabah \\ *Corresponding author E-mail: Soon.Eng@uniten.edu.my
}

\begin{abstract}
Remote sensing using drone or UAV (unmanned aerial vehicle) is the current trends and this technology can provide unrevealed lifealtering benefits to mankind. Drones are being used in many sectors such as for military, research, agricultural and recreational means. This technology not only can reduce the time of inspection, but it is also giving many benefits such as provides real-time live video for site inspection that can help user to analyze site logistic and speeding up the overall tasks. However, vegetation monitoring using remote sensing has its own challenges in terms of processing the captured image and data. Somehow, previous research has suggested a lot of different possible algorithm that could be used for post-processing the data gathered. Nevertheless, most of the algorithm requires a specific sensor in order to get the result. The objective of this paper is to identify and verify the algorithm that is suitable to process the vegetation image. This research will use the data gathered from various area by using consumer camera and process by using Visible Atmospherically Resistant Index (VARI) indices. Finally, this research will observe the accuracy of the result analyzed using the VARI and identify the characteristic of the algorithm.
\end{abstract}

Keywords: vegetation monitoring; remote sensing; Visible Atmospherically Resistant Index; unmanned aerial vehicle; drone;

\section{Introduction}

Planting vegetation brings lots of benefits to human and the environment such as reducing the temperature within urban area [1][2]. However, the vegetation must be monitored and maintained as to avoid any hazards that could harm humans. For example, vegetation that is not maintained by power agency could bring harmful effects towards power lines and towers.

According to a study, there is about 10 percent of trees that are planted in a private property that effects the power cable [2]. Additionally, another study mentioned that a developer in Bakersfield, CA planted 300 red weed trees directly under power cables and decline to remove those trees despite it affect the power cable trees [2].

To overcome these problem, monitoring activities have been carried out by inspecting vegetation near power cable and tower on regular basis. It was stated that the total cost for monitoring those vegetation requires between 2 billion to 10 billion dollars per year, however that cost does not include vegetation trimming [3].

Dr. B. Don Russel et al. have conducted an experiment in verifying that tree branch could cause power cable failures and power outage when having contact with the power cables within the area. [4]

From the outcome of the experiment, it has proved that when a tree branch has a contact with a power cable structure would have the highest possibility to cause a power failure. Additionally, it took approximately 3 minutes to start a fire when a tree branch have a contact with the cables. The experiment get into approximately 4 minutes, a sign of electric power that goes through the lit up tree branch begin. The experiment also proved that it would not only cause power outage but at the same time, it also brings dangerous accidents towards the public and within the area especially an area that is nearby a forest. These incidents would also cause large expenses in single maintenance to patrol, inspecting, cutting and managing vegetation.

Apart from that, the lack of awareness among planters and developers of vegetation is one of the contributors to these incidents to happen. Without proper monitoring and management, the possibility for the incidents to happen is higher. Since then, few countries have established guidelines in order to reduce the risk of vegetation being planted near the power tower and cable [4].

\section{Problem Statement}

In order to overcome the incident mentioned above, monitoring activities have been carried out by monitoring and inspecting vegetation near power cable and tower. A research by Rancea and George $\mathrm{V}$ stated the cost of monitoring those vegetation is between 2 billion to 10 billion dollars per year, however that cost does not include vegetation trimming [3]. Besides, according to the SA power networks mentioned that their method of inspection vegetation is by using a four-wheel drive (4WD) vehicle to get to the location and traverse along every single line of vegetation that is close to the power cable. Once required to perform tree cutting, tree cutting crew will be assigned to perform vegetation clearance within the area [5].

In addition, a traditional method of inspection is to send a welltrained worker on-side [6]. With this, the activities required a lot of time and man-power for the inspection task.

\section{Literature Review}

This section discusses about the existing monitoring methods to reduce the accident from happening. 


\subsection{Vegetation Planting Near Power Line Guideline}

Due to unexpected incidents that constantly happened, a few countries' related agencies have released their own guidelines to refer and to ensure that the incident is able to controlled.

According to guideline produced by National Grid Australia, an energy company that covers Massachusetts, New York, Rhode Island and United Kingdom (UK) [7]. National Grid will scheduled a tree trimming every five to seven years. Additionally, National Grid will only hire a certified contractor to perform trimming action with "directional pruning" technique.

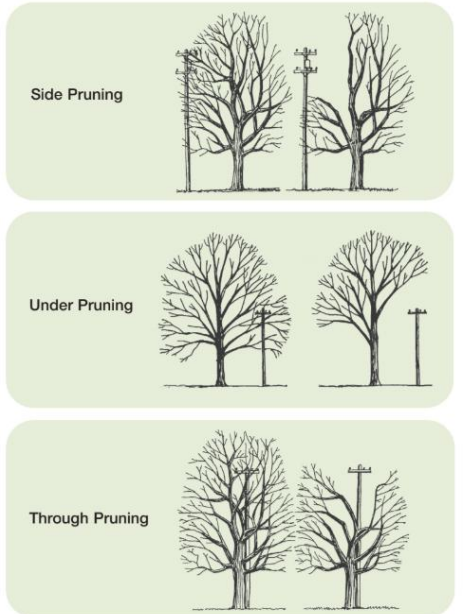

Fig. 1: Pruning method being used by Nasional Grid

Based on the guideline, the trees will be maintained for a minimum of ten to fifteen feet away from the power lines. As presented by Figure 1, there are three ways of pruning that are side pruning, under pruning and through pruning. Each method consists of different ways to maintain the height of the trees [7].

Second is the guideline from Government of Western Australia named as "Guidelines for the management of vegetation near power lines". In their guideline clearly stated the voltage for distribution minimum of 240 volts to highest is 33,000 volts and for transmission minimum 66,000 volts to 330,000 volts. However, the clearance area is required to be at 2 meters or onwards between power lines and vegetation [8].

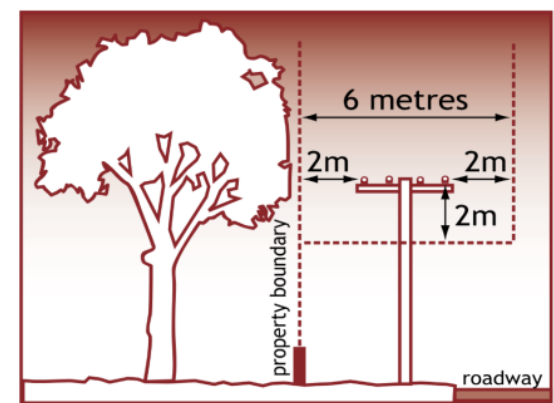

Fig. 2: Vegetation clearance zones in urban areas from Western Australia Guideline [8]

Figure 2 shows a diagram of the maintain clearance area between power line and vegetation or tree.

Next, a protocol for vegetation management near powerlines for 2016 to 2018 was published by SA power networks. SA Power Networks is the operator of distribution power supply in South Australia [5]. According to the guideline, an annual cyclic program will be carried out for high risk and medium risk bushfire. While non-bush fire risk, a three year of cyclic program will be carried out.

The interesting part of the guideline is they have four new techniques to be used in the pruning program. The first technique is formative pruning. Whereby selective actions will be used in taking out specific branches to enhance form and improve structure. The second technique is selective pruning. This method is to remove any branches that would likely to cause specific problems. Next, the pollarding technique is to remove the pollard head of bud and vigorous shoots. Lastly, the tree removal technique will be used depending on the tree's visual, health and any related safety reasons.

\subsection{Vegetation Monitoring by Using Technology}

While technology keeps involving, a few technologies is available for vegetation monitoring. At the same time, lots of researchers found that their traditionally monitoring methods are being replaced with the latest technology.

This is in line with research done by Lawesson, J.E. et al. about their study related to vegetation studies and monitoring in Nordic countries. A computer program named as Geographic Information System or shortly known as "GIS". This system was developed to gather the data and use it to describe the places on the earth's surfaces. Apart from that, the system also uses the data to manipulate and analyze the spatially related data. Additionally, GIS also capable in showing the distribution of species, plots or vegetation by using descriptive modeling and prescriptive modeling [9].

Another research highlighted that aerial remote sensing techniques are used to detect and classify the vegetation by using a developed algorithm that uses spectral band ratio as an input to a pulse coupled neural network and morphological reconstruction. However, their algorithm could only achieved $75.2 \%$ of the segmentation accuracy, while $96 \%$ are detection rate. This is because undersegmentation of tree clusters and crown trees are very sparse and presented low contract to the background. To improve the trees segmentation, the researchers require data that is captured by Light Detection and Ranging (LiDAR) to support and provide density of points. In result, the vegetation has a distinctive spectral signature and characterized by a low reflectance in the visible part of the solar spectrum [10].

Besides, another research found that the normalized difference vegetation index (NDVI) can be use to identify the vegetation's health. It is the combination of Red, Green and Blue (RGB) images with green biomass data to produce the result of the plantation's health. Furthermore, in their research, they have also used image obtained from Landsat Thematic Mapper (TM) imagery. Consequently, a formulation to calculate are applied to produce the end results value of the vegetation. Lastly, they compared the changes of the vegetation. The conclusion of the research claims $85 \%$ of the accuracy are from NDVI method [11].

Other than that, a study about satellite vegetation greenness by using satellite data. In this research, the data was gathered from Advanced Very High Resolution Radiometer (AVHRR) sensor on board on NOAA afternoon satellite. The numeric value will be recorded to represent the amount of light reflected from the surface of the earth. By using the data gathered, the researcher used NDVI formula to calculate the result. However, the researcher mentioned they excluded the data that could be cloudy and snow because it could affect the final value. To ensure the accuracy of the result, any data that shows cloudy and snow was excluded from the final value. [12].

Lastly, another research that was conducted in India proved by using free data from NOAA \& TERRA satellite sensor and open source concept monitoring of vegetation tools able to achieve near real time [13]. The researcher gathered daily data of the studied area from geographical information system (GIS) software named Quantum GIS. Then again, using the NDVI formula to convert the data into identifiable healthy vegetation within the map. Then, the NDVI map will be converted again into the image and uploaded to the server to allow the end-user to view the NDVI result through internet browser.

In conclusion, every method or technique mentioned above has its own advantage and disadvantage. As for satellite image as data 
source can remove the time to conduct the on-site inspection and no longer need an expert to perform the task anymore, but according to a research claim that satellite image or data is lack of capability to capture enough details of ground objects due to the coarse ground resolution [14]. To add, another research claimed that satellite image or data takes at least two weeks to reach to the user [15]. Lastly, remote sensing can capture the latest image or data by the user itself when needed. However, the limitation of the weight of the payload or sensor requires carrying of the unmanned aerial vehicle (UAV) and period of flying around the inspection area [10][16].

\section{Equipment and Processing}

\subsection{Consumer Digital Camera}

There are many type of digital camera for capturing photos and videos. The camera can be divided into simple basic camera for beginner and advanced camera such as digital single lens reflex (DSLR) type of camera. For better feature camera that are able to capture from very high angle that normally human unable to make it, drone produced from DJI will be the greatest choice. DJI drone with built-in camera and its features almost similar to digital camera is powerful in capturing certain angel that is quite hard to reach or capture the photo.

According to a research by Lebourgeois et al., most of the digital cameras capture images in JPEG or TIFF formats with the Red, Green and Blue (RGB) channels. Those RGB channels are obtained through simple image processing method. RGB channels also can be used for image classification or combined in spectral indices to be correlated with the surface parameters of interest [17]. Furthermore, they found that some of the camera produce two type of images. Instead of having JPEG format, another type of image will be recorded in RAW format known as the unprocessed digital photo. The difference between these two formats is the value of depth. Whereby JPEG format used 8 bits, while, the RAW format is using 12 bits. This means the RAW (Unprocessed) format will use slightly higher value depth compared to JPEG. To conclude, the RAW format processed the vegetation image in high quality compared to JPEG format. As the JPEG format compressed the produced image in low quality and the digital number is not linear with the brightness of the scene. [17].

After understanding the research above, we found that the camera type that is able to produce RAW format mostly is DSLR and some of the entry-type cameras have a feature to capture image in RAW format. However due to the image or data gathering stage found most are in JPEG format, we decided to proceed with the I JPEG format to perform the processing stage.

\subsection{Post-Processing}

In this stage, an open-source geographic information system (GIS) software named "QGIS" is used. QGIS was licensed under the GNU General public license and an official project of the Open Source Geospatial Foundation (OSGeo). In addition, QGIS consist a user-friendly interface and that allows users to install plug-in from other developers into the software to run others process.

According to a research, Visible Atmospherically Resistant Index (VARI) can minimize the sensitivity of the atmoshpheric effects and allows estimation of the vegetation fraction [18]. Based on the formula of VARI only required the red, green and blue (RGB) band only to produce the result.

$\mathrm{VARI}=\left(\mathrm{R}_{\text {Green }}-\mathrm{R}_{\mathrm{Red}}\right) /\left(\mathrm{R}_{\text {Green }}+\mathrm{R}_{\mathrm{Red}}-\mathrm{R}_{\text {Blue }}\right)$

Based on the formula above, RGreen will refer to the data set of the green band of the image. Secondly, RRed is referring to the data set of the red band of the image. Lastly, RBlue is referrig to the data set of the blue band of the image. The reason of choosing
VARI instead of NDVI is that based on previous research, NDVI requires near-infrared band (NIR) image to perform calculation. Thus, VARI is more suitable formula to use for this experiment.

$\mathrm{NDVI}=(\mathrm{NIR}-\mathrm{Red}) /(\mathrm{NIR}+\mathrm{Red})$

$[11][12][13]$

After the image being processed, the result will be produced in TIFF format. Meanwhile, the image will show as grey band format only (Fig. 3). After that, the result image will be imported again into the QGIS and change the image display by using singleband pseudocolor (Fig. 4).

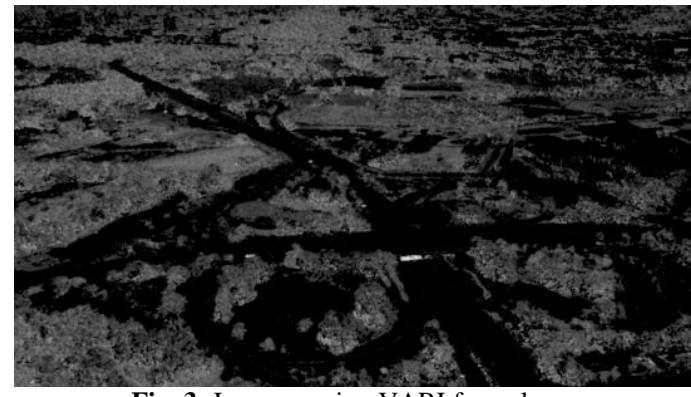

Fig. 3: Image - using VARI formula

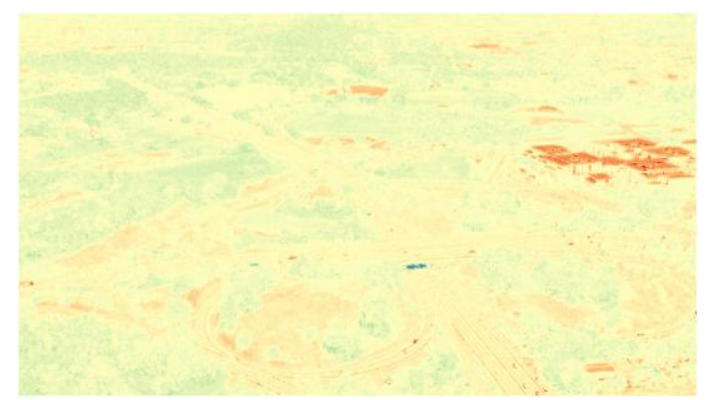

Fig. 4: Image - using VARI formula with Singleband pseudocode

Finally, to identify the vegetation clearly, the colour that symbolizes non-vegetation will be removed to improve the visibility of the tree in the picture.

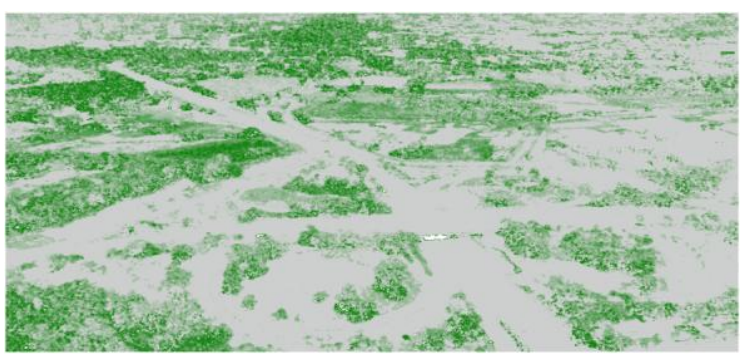

Fig. 5: Image - using VARI formula

\section{Result and Discussion}

We did not run all the data gathered, but we use the selected data only. The figure below is the result of the image.

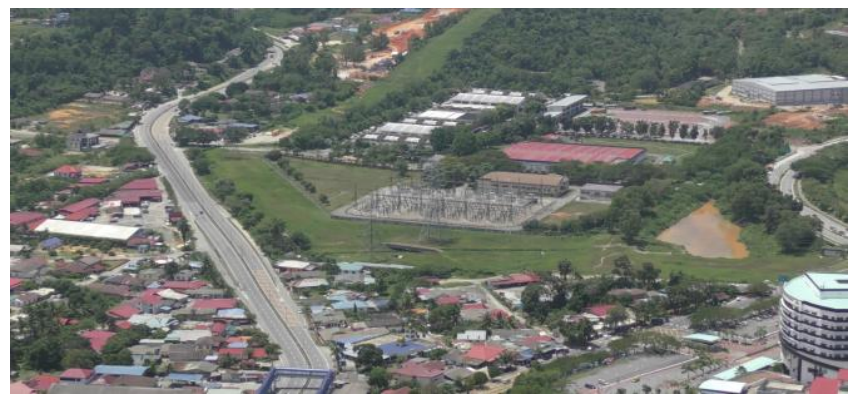

Fig. 6: Sample image capture by drone 


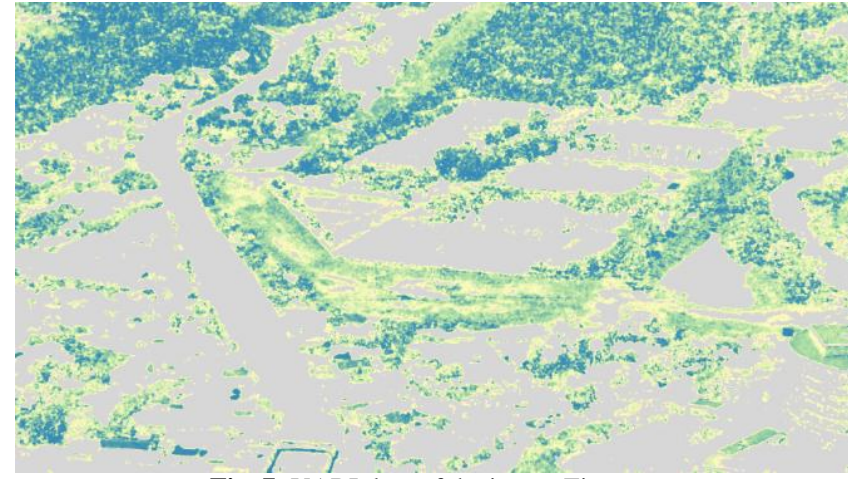

Fig. 7: VARI data of the image Figure 6

Figure 6 is an image of a city with some main road and village houses around the city. While on the top right is where the industry area located that are surrounded with vegetation. The VARI result showed in Figure 7 proved that most of the vegetation been highlighted in the image. The dataset of the result with the minimum value of -1.25 and the maximum value of 0.9145

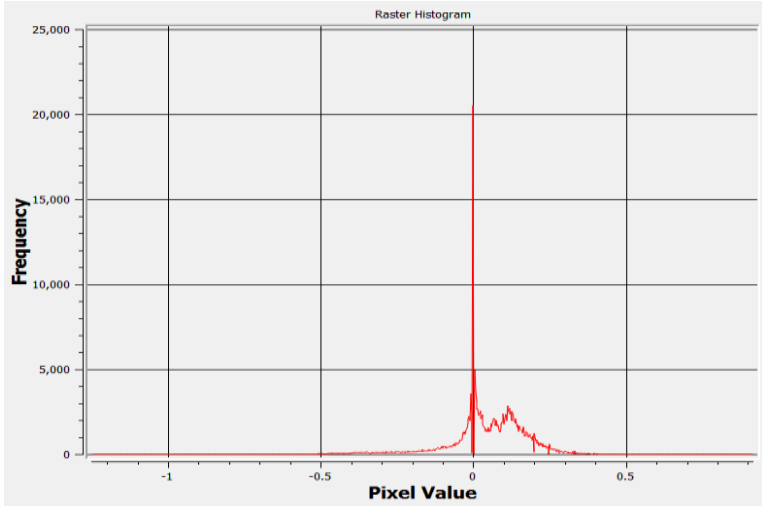

Fig. 8: Raster histogram of VARI data of image Fig. 6

The historgram above (Fig. 8) was generated by qGIS on the VARI datasaet. It proves that most of the pixel value from the image figure 6 is around zero and above zero. This is due to the vegetation found from the image is slightly more than the nonvegetation.

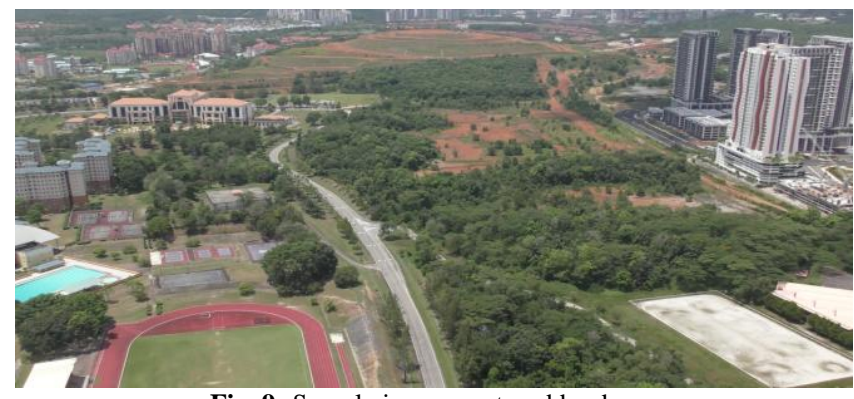

Fig. 9: Sample image captured by drone

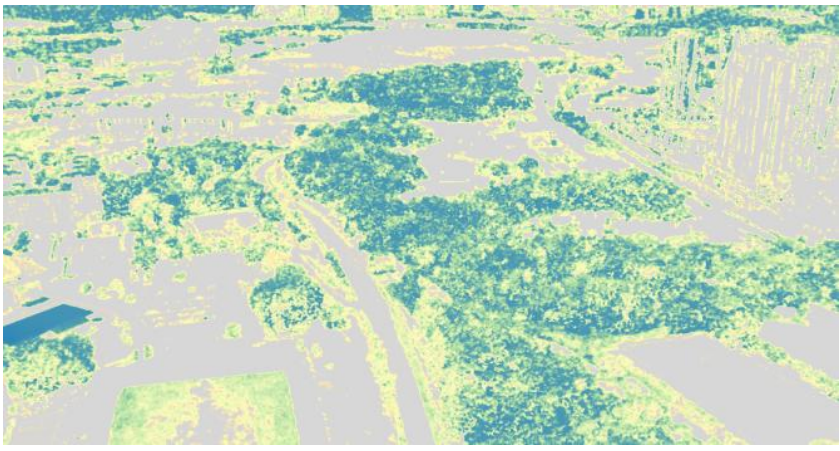

Fig. 10: VARI data of the image Figure 9
Next, Figure 9 above is a view of the University Tenaga Nasional (UNITEN). The sample image captured some part of the apartment building, soccer field, swimming pool and small vegetation forest. In result, figure 10 presents the VARI result with the vegetation highlighted in green. The dataset of the result consist a minimum value of -1 and the maximum value of 1.30538. The main highlight of this result can be the area of small vegetation forest. Not only it shows the large area of trees but at the same time, it shows some part of the area that consist red sand or less vegetation.

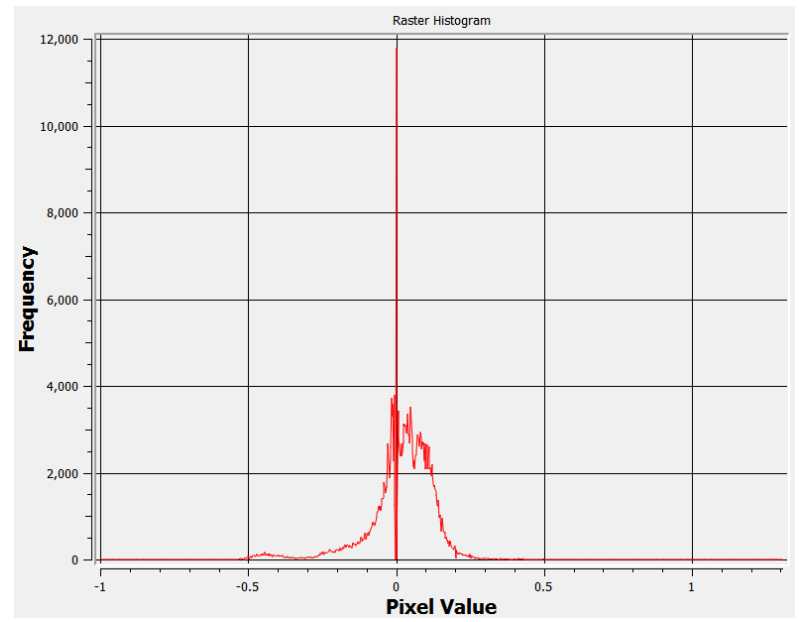

Fig. 11: Raster histogram of VARI data of image Fig. 9

Lastly is the raster histogram of the VARI image for figure 9 sample image, Similarly with image in Figure 6, it consists pixel value that is around zero

However, there is also some lack of accuracy with some image captured from the urban or city area because some object such as green colour object, swimming pool area detected as positive VARI value. Figure below show the example of the non-accuracy area.

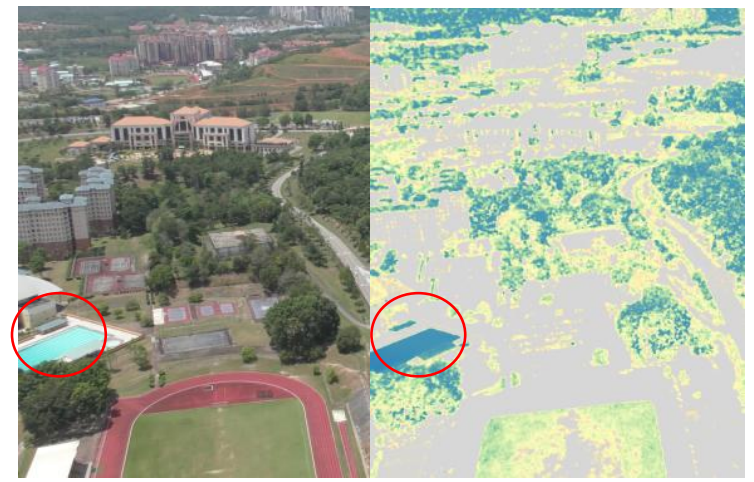

Fig. 12: Inaccurate detection sample image

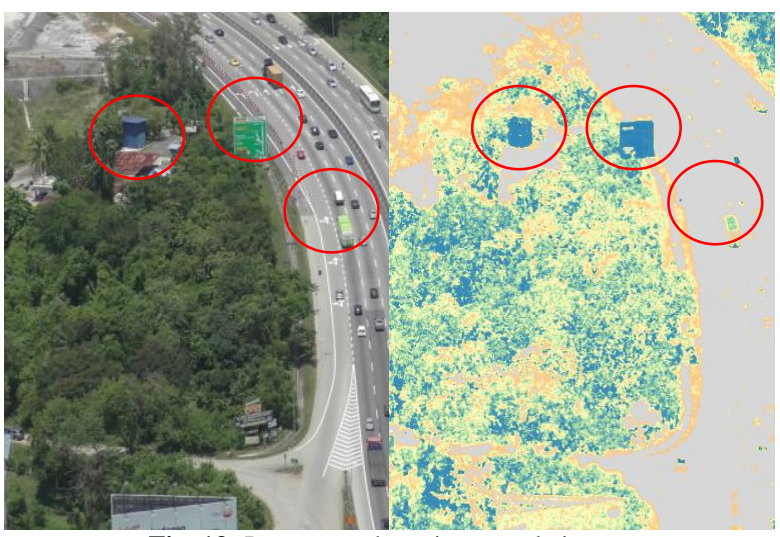

Fig. 13: Inaccurate detection sample image 
The figure above shows the areas of the inaccurate detection of vegetation, this is due to the color of the object that are closed within the vegetation range.

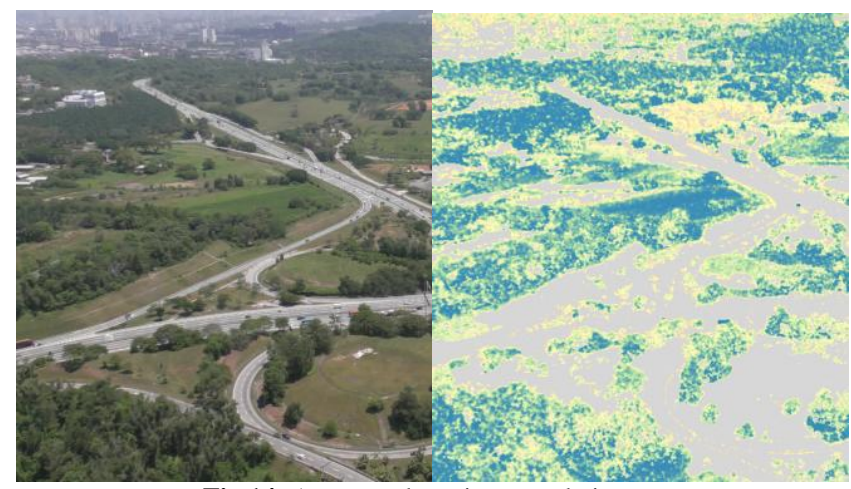

Fig 14: Accurate detection sample image

Fig. 14 shows an example of urban area image. The result clearly indentified green area and road area. However, some part of the grass area was given a negative value. This means that the VARI formula is unable to detect the grass well in some situation.

The result proves that vegetation monitoring by using simgle formula like VARI is good to detect vegetation and nonvegetation area. However in certain cases there are still some inaccuracy of image detected. We suggested that another formula such as Green Leaf Index (GLI) and Normalized Difference Vegetation Index (NDVI). This might increase the vegetation detection accuracy rate higher with two phases of verification by comparing the result of different formula.

\section{Conclusion}

As a conclusion, the results after image has been processed using the formula shows that value for detected vegetation are in the range of 0.01 or above.

The formula is able to identify the vegetation and the health very accurate if the image is not captured from urban or city area with many objects that has color that closes to the vegetation. In the future works, another formula and algorithm will be identified to test its accuracy and reliability for the consumers with minimal cost.

\section{Acknowledgement}

This research was supported by UNITEN Internal grant Project code J510050744. We acknowledged the use of facilities and equipment provided by MMC Drone and Kembara Impian Technologies Sdn. Bhd. We would wish thank to Tuan Hussin Daud. and Mr. Alan from Kembara Impian and Mr. Edison and Mr. Ken from MMC Drone for providing us suggestion and idea based on their expertise.

\section{References}

[1] Pauline, L., Damien, P., \& François, C. (2014). The benefits of urban vegetation, (February).

[2] William, Brock, M., \& Weissman, S. (2012). Trees and Power Lines: Minimizing Conflicts between Electric Power Infrastructure and the Urban Forest.

[3] Rancea, G. V. (2014). Evaluation of Methods for Control of Vegetation in Utility Corridors.

[4] Reducing faults created by vegetation contact on utility lines. (n.d.) Retrieved April 19, 2018, fromhttps://phys.org/news/2010-11faults-vegetation-contact-lines.html

[5] SA Power Network (2016). 'Protocol for vegetation management near powerlines 2016-2018.' Retrieved from https://www.sapowernetworks.com.au/public/download.jsp?id=549 55

[6] Qayyum, A., Malik, A. S., Mohamad Saad, M. N., Iqbal, M., Ahmad, R. F., Tuan Abdullah, T. A. R. B., \& Ramli, A. Q. (2015). Monitoring of vegetation near power lines based on dynamic programming using satellite stereo images. 2014 IEEE International Conference on Smart Instrumentation, Measurement and Applications, ICSIMA 2014, (November), 25-27. https://doi.org/10.1109/ICSIMA.2014.7047429

[7] National Grid (2009). 'What you should know about trees and your electric service.' Retrieved from https://www9.nationalgridus.com/non_html/Important_Info_About _Trees_and_Electric_Service_NE.pdf

[8] Government of Western Australia (2012). 'Guidelines for the management of vegetation near power lines.' Retrieved from https://www.commerce.wa.gov.au/sites/default/files/atoms/files/veg etation_powerlines_guidelines.pdf

[9] Lawesson, J. E. (Editor) ; Eilertsen, O. ; Diekmann, M. ; Reinikainen, A. ; Gunnlaugsdóttir, E. ; Fosaa, A. M. ; Carøe, I. ; Skov, F. ; Groom, G. ; Økland, T. ; Økland, R. ; Andersen, P. N. ; Bakkestuen, V./ A Concept for Vegetation Studies and Monitoring in the Nordic Countries. Copenhagen: Nordic Councils of Ministers, 2000. 125 p. (TemaNord, Vol. 2000:517).

[10] Li, Z., Walker, R., Hayward, R., \& Mejias, L. (2010). Advances in Vegetation Management for Power Line Corridor Monitoring Using Aerial Remote Sensing Techniques. Proceedings of the First International Conference on Applied Robotics for the Power Industry (CARPI), 1-6. https://doi.org/10.1109/CARPI.2010.5624431

[11] Hayes, D. J., \& Sader, S. a. (2001). Comparison of Change Detection Techniques for Monitoring Tropical Forest Clearing and Vegetation Regrowth in a Time Series. Photogrammetric Engineering \& Remote Sensing, 67, No. 9, 1067-1075. https://doi.org/citeulikearticle-id:7954186

[12] Burgan, R. E., \& Hartford, R. A. (1993). Greenness With Satellite Data, (May).

[13] Gopinath, G. (2015). Free data and Open Source Concept for Near Real Time Monitoring of Vegetation Health of Northern Kerala, India. Aquatic Procedia, 4(Icwrcoe), 1461-1468. https://doi.org/10.1016/j.aqpro.2015.02.189

[14] Feng, Q., Liu, J., \& Gong, J. (2015). UAV Remote sensing for urban vegetation mapping using random forest and texture analysis. Remote Sensing, 7(1), 1074-1094. https://doi.org/10.3390/rs70101074

[15] Sugiura, R., Noguchi, N., \& Ishii, K. (2005). Remote-sensing technology for vegetation monitoring using an unmanned helicopter. Biosystems $\quad$ Engineering, 90(4), 369-379. https://doi.org/10.1016/j.biosystemseng.2004.12.011

[16] Suzuki, T., \& Amano, Y. (2009). Development of Low-Cost and Flexible Vegetation Monitoring System Using Small Unmanned Aerial Vehicle. Iccas-Sice, 2009, 67, 4808-4812.

[17] Lebourgeois, V., Bégué, A., Labbé, S., Mallavan, B., Prévot, L., \& Roux, B. (2008). Can commercial digital cameras be used as multispectral sensors? A crop monitoring test. Sensors, 8(11), 7300 7322. https://doi.org/10.3390/s8117300

[18] Gitelson, A. A., Kaufman, Y. J., Stark, R., \& Rundquist, D. (2002). Novel algorithms for remote estimation of vegetation fraction. $R e-$ mote Sensing of Environment, 80(1), 76-87. https://doi.org/10.1016/S0034-4257(01)00289-9 